\title{
Liderazgo con Empowerment: Promotor de la innovación
}

\author{
Petit Torres, Elsa Emilia* \\ Gutiérrez González, Lorena Beatriz ${ }^{* \star}$
}

\section{Resumen}

En este artículo se describe el Modelo de Liderazgo con Empowerment para identificar su perfil promotor de la innovación en las organizaciones empresariales. Se adoptó una estrategia de investigación cualitativa basada en la aplicación del método interpretativo, propuesto por Joseph Kockelmans (1975), el cual se fundamenta en la técnica de análisis de contenido de artículos especializados. Se describe el Modelo de Liderazgo Empowerment como promotor de la innovación bajo la consideración de las siguientes características: 1. Cultura Empowerment con valores innovativos, 2. Confianza y compromiso hacia la organización empresarial, 3. La intrategia como instrumento para la unidad, 4. El locus de control interno para impulsar el cambio continuo, 5. Mantener la competencia y 6. Optimismo, creatividad y colaboración cruzada. Se concluye que un punto de encuentro conceptual entre la Teoría de la Innovación, el Enfoque de la Gestión del Conocimiento y la Teoría Gerencial, consiste en asumir al liderazgo como un determinante de la innovación. La Teoría Gerencial en el marco de la Gestión del Conocimiento y el Modelo de Liderazgo Empowerment, explica conceptualmente cómo es posible aprovechar las ideas explotando recursos, generando capacidades y desarrollando competencias en la organización a partir de las aportaciones de todos sus miembros, centrándose en el valor de uso de las ideas, lo cual constituye el eslabón que permite relacionar las variables "Liderazgo Empowerment" y "proceso de innovación".

Palabras clave: Liderazgo con Empowerment, Innovación, organizaciones empresariales.

Recibido: 12-09-05. Aceptado: 05-05-07

Universidad del Zulia. Facultad Experimental de Ciencias. Departamento de Ciencias Humanas. Unidad Académica Estudios del Desarrollo Apartado 526. Maracaibo-Venezuela.

E-mails: eept2002@hotmail.com - Igutierrez73@hotmail.com

** Universidad del Zulia. Facultad Experimental de Ciencias. Departamento de Ciencias Humanas. Unidad Académica Estudios del Desarrollo Apartado 526. Maracaibo-Venezuela.

E-mail: Igutierrez73@hotmail.com 


\section{Empowerment Leadership: Promoter of Innovation}

\section{Abstract}

This article describes the model of leadership with empowerment in order to identify its profile as a promoter of innovation in business organizations. A strategy of qualitative investigation was adopted, based on application of the interpretative method proposed by Joseph Kockelmans (1975), which is founded in the technique of analyzing the contents of specialized articles. The empowerment leadership model is described as a promoter of the innovation considering the following characteristics: 1 . Cultural empowerment with innovative values; 2 . Confidence and commitment to the business organization; 3. intrategia as an instrument for unity; 4. Locus of internal control to impel continuous change, 5. maintain competition and 6. optimism, creativity and cross-collaboration. Conclusions are that a conceptual contact point among the theory of innovation, the knowledge management approach and management theory consists of assuming leadership as a determinant of innovation. Management theory within the framework of knowledge management and the empowerment leadership model, explains conceptually how it is possible to take advantage of ideas, exploit resources, generate capacities and develop competences in an organization based on the contributions of all its members, centering on the value of using ideas, which constitutes the link that permits relating the variables "empowerment leadership" and the "process of innovation."

Key words: Empowerment leadership, innovation, business organizations.

\section{Introducción}

La innovación constituye una variable que influye en el crecimiento, desarrollo y competitividad de los países y organizaciones empresariales. Ésta ha sido estudiada teóricamente por economistas tales como Schumpeter (1944), Usher (1955), Schmookler (1962), Rosenberg (1979), Nelson y Winter (1982) y Pérez (1989), entre otros. Dichos autores afirman que el fenómeno de la innovación surge en el seno empresarial con fines netamente comerciales y con implicaciones sociales. Sin embargo, el proceso de innovación, no se ha trabajado suficientemente desde la perspectiva de la teoría organizacional, considerando el liderazgo como elemento inspirador de dicho fenómeno.
El enfoque teórico de la innovación propio de los países desarrollados, resalta el tema de la racionalidad económica y técnica más no hace hincapié en la racionalidad gerencial, dejando de lado la oportunidad de reforzar el enfoque teórico de la innovación desde la perspectiva estratégica y organizacional, lo que significa que en la discusión teórica no se destaca al liderazgo como factor determinante en la generación de innovaciones.

Considerar esta limitación teórica, implica resolver un problema conceptual que permite analizar el liderazgo organizacional con Empowerment como un determinante de la innovación. Los resultados de este estudio contribuyen a fortalecer la Teoría de la Innovación y a promover la dinámica del proceso de innovación 
en los países y en las organizaciones empresariales para que estas asuman un rol protagónico en la creación y desarrollo de capacidades para innovar o generar valor, sobre todo en los países en vías de desarrollo.

Para que un país ó una organización empresarial se conviertan en foco de innovación, es necesario estimular el proceso inventivo en sus miembros. El proceso inventivo, de acuerdo a Schmookler (cit. Por García, L., 1989: 29-30) se basa en la generación de ideas, que pueden convertirse en cambios no triviales o innovaciones difundibles tecnológicamente. La invención, como base del proceso innovador, requiere de estímulo gerencial mediante un liderazgo efectivo que promueva actos creativos en los procesos que se ejecutan en todas las estructuras productivas de los países.

Un liderazgo que propicia el proceso de innovación en su organización, es aquel que fomenta la iniciativa y la motivación de sus miembros, aumentando su participación, cultura y formación en pro del desarrollo y competitividad de la organización. Todo esto va apareado con una estructura más nueva, más plana y más flexible constituida por equipos de liderazgo inter actuantes ligados por el conocimiento y por las redes de comunicación, con responsabilidad descentralizada y participación del poder, todas estas, características que describen la naturaleza del Liderazgo con Empowerment.

En concordancia con estas características ha surgido el Empowerment, como nuevo modelo para el liderazgo, cuyo enfoque consiste en dar poder o liberar el potencial de la gente, otorgando a todas las personas roles de liderazgo de manera que puedan contribuir con lo mejor de sus capacidades (Quigley, 1997; McFarland, Senn, Childress, 1996: 61-64).

El Liderazgo con Empowerment también es dar y adquirir continua y permanentemente el poder del conocimiento. Poder que dentro de una estructura organizacional plana y flexible puede ser compartido de forma dinámica para detonar el surgimiento de las innovaciones en sus diferentes grados de novedad (incremental o radical) y naturalezas (en procesos, en productos, organizacionales, en servicios, comercial, tecnológica, por sectores industriales y para el desarrollo local). Es por tal razón, que se plantea la relación conceptual entre Liderazgo con Empowerment e innovación.

Con base en lo anterior surge la siguiente interrogante de investigación: ¿Qué características del Modelo de Liderazgo con Empowermentcontribuyen a fomentar el proceso de innovación en las organizaciones empresariales? En tal sentido, el objetivo general de este artículo se orienta hacia la descripción del Modelo de Liderazgo con Empowerment para identificar su perfil promotor de la innovación en las organizaciones empresariales.

\section{Metodología}

Para describir el Modelo de Liderazgo con Empowerment e identificar su carácter promotor de la innovación en las organizaciones empresariales se adoptó una estrategia de investigación cualitativa basada en la aplicación del método interpretativo, propuesto por Joseph Kockelmans (1975).

El método propuesto, se fundamenta en la técnica de análisis de conte- 
nido, realizada en función de cuatro criterios principales: 1) Ubicación y revisión de artículos especializados, 2) Categorización de sus contenidos, 3) Explicación y ubicación de cada contenido dentro de la estructura del trabajo, y 4) Identificación de lo esencial de cada contenido para dar respuesta a la interrogante que sistematiza el problema de investigación.

\section{Fundamentación Teórica}

Para dar cumplimiento al objetivo de este artículo, el enfoque teórico más apropiado para el análisis de los resultados es el de la "Gestión del Conocimiento". Éste tipo de administración es producto de la evolución en la disciplina de la Gestión Tecnológica (INOVA, 2003), surge a mediados de la década de los años noventa y ha prevalecido hasta hoy en las organizaciones, empresas e inclusos individuos que buscan el progreso y una mejor calidad de vida.

La Gerencia del Conocimiento procura gestionar el conocimiento de manera colaborativa, creando un sistema total de innovación con la participación y la auto motivación de todos los actores existentes dentro de la cadena productiva incluyendo la sociedad y los clientes.

De acuerdo a Caires (2003), los gerentes del conocimiento se preocupan por las necesidades de conocimiento de la empresa. Ellos se encargan de realizar la investigación para determinar que conocimiento es necesario para crear decisiones y permitir las acciones. Los gerentes de este proceso monitorean el flujo de conocimientos, y el desempeño es evaluado en términos de activos intelectuales o de conocimientos y la habilidad de crear y aplicar nuevas ideas en el mercado. La creación de redes de aprendizaje, y formulación de estrategias también son parte de este sistema.

El sistema de gestión de conocimiento posee las características de manejar diferentes inputs y producir varios outputs, dentro de diferentes contextos y experiencias siendo fácilmente ajustadas y actualizadas dentro de una base de conocimiento.

Desde la perspectiva teórica de la gestión del conocimiento es posible describir las características del Modelo de Liderazgo con Empowermentcomo promotor de la innovación para establecer una relación conceptual entre el Liderazgo con Empowermenty sus efectos sobre la dinámica del proceso de innovación, dicha relación se sustenta en la naturaleza colaborativa como se gestiona el conocimiento, proceso en el que participan auto motivados todos los miembros de una organización empresarial.

\section{El Liderazgo con Empowerment como Promotor del Proceso de Innovación en la Organización Empresarial}

A continuación se describen las principales características del Modelo del Liderazgo con Empowerment como promotor del proceso de innovación en organizaciones empresariales:

1. Cultura Empowerment con valores innovativos

Conforme a McFarland, Senn y Childress (1996:86) "La esencia del Empowerment reside en la mente de los líderes, que constantemente estimulan el surgimiento de las nuevas ideas y la inno- 
vación en toda la organización." Del mismo modo, los autores destacan que "La innovación no tendrá lugar hasta tanto la cultura como un todo no provea Empowerment y nutra las nuevas ideas." No obstante, si la organización no tiene internalizado el valor innovación en su cultura, difícilmente ocurrirá el proceso de innovación.

Cuando una organización es liderada bajo el enfoque Empowerment reforzando el valor innovación en su cultura, se moviliza a los individuos y a los equipos autodirigidos no sólo a ejecutar órdenes, sino también a generar nuevas ideas mejorar procesos, productos y servicios, con resultados positivos en el crecimiento y desarrollo competitivo de la organización.

En este contexto, muchos líderes temen que si permiten mayor laxitud, van a perder el control; pero la ironía de todo esto consiste en que a medida que "pierden el control", logran que más gente de todos los niveles aporte ideas y se desempeñen responsablemente en relación con la calidad de los productos y el servicio al cliente. En una cultura empresarial saludable, que tenga un liderazgo con $\mathrm{Em}$ powerment, la gente sentirá que no sólo se escucharán sus ideas sino, más importante aún, que se pondrán en ejecución (McFarland, Senn y Childress 1996:87).

2. Confianza y compromiso hacia la organización empresarial

Existen evidencias empíricas (Pfeffer, 1998; cit por Cardona, 2001:19) donde se ha comprobado que las compañías pueden obtener mucho más beneficios (superiores en más del 30\%) si incorporan prácticas directivas que incrementen el compromiso y la competencia de su gente. Este resultado es válido para muy diferentes tipos de industrias desde aceros a semiconductores, -para gran variedad de compañías- desde empresas manufactureras a organizaciones de servicios.

De acuerdo con Cardona (2001:30) la confianza y el compromiso de las personas con la empresa son condición necesaria para los procesos de innovación y adaptación que caracterizan a las empresas exitosas.

En tal sentido, conviene destacar la existencia de estudios (de Geus, 1997; cit por Cardona, 2001:19), donde se demuestra que las compañías que han sobrevivido más de un siglo desarrollan ciertas características comunes al crear una comunidad de directivos y empleados comprometidos. Las empresas de larga vida han sido capaces de adaptarse a cambios importantes, e incluso radicales, en el entorno político, social y económico.

Estos estudios revelan de modo claro y sistemático que tanto el rendimiento de la empresa como su supervivencia a largo plazo dependen, en gran medida, del tipo y profundidad del compromiso de sus miembros con la misma. Cada vez es más necesario contar con gente comprometida para competir con éxito en un entorno globalizado.

La profundidad del compromiso de los miembros con su organización, es medida por el grado de identificación que éstos tengan con la Visión de la empresa, cuando todos los miembros de la organización comparten de manera consensuada los elementos que constituyen la Visión (valores, misión, metas, estrategias, tácticas y responsabilidades) entonces es posible observar que los miembros se 
involucran en los procesos, direccionando sus esfuerzos para alcanzar sus objetivos comunes.

3. La intratégia como instrumento para la unidad

Conforme a Cardona (2001:28), las intrategias son prácticas directivas que han incrementado el nivel de unidad en diversas empresas. Estas intrategias, si están bien alineadas con la estrategia de la compañía, aumentan la capacidad de la misma para conseguir un alto rendimiento y una larga vida. Pfeffer (1998; cit por Cardona, 2001:28) recoge una lista de siete de estas prácticas: seguridad de empleo, contratación selectiva, descentralización de toma de decisiones, compensación contingente, formación extensiva, pocas diferencias de status, y amplia información financiera y de resultados. Este autor advierte que, para que éstas prácticas tengan éxito, necesitan ser, en primer lugar, internamente consistentes una con otra y en segundo lugar, externamente consistente con la estrategia.

En tal sentido y de acuerdo a Cardona (2001:30), del mismo modo que la estrategia mira el impacto económico de las decisiones directivas, la intratégia mira como las decisiones directivas afectan el nivel de unidad de la compañía. Debido a que las decisiones directivas tienen impacto no sólo en el resultado económico ó beneficio, sino también en el resultado organizativo ó unidad, los directivos deberían evaluar sus decisiones desde ambos puntos de vista para así, mantener su posición competitiva.

La innovación tiene un carácter estocástico, es decir, no es posible pre- decir cuándo ocurre; no obstante, desde la perspectiva gerencial es factible crear las condiciones organizacionales para su surgimiento mediante el diseño de mecanismos y formas organizacionales que permitan la interacción sinérgica de sus miembros con la finalidad de producir nuevas ideas mediante el desarrollo del poder de las relaciones; por ejemplo, el trabajo en equipo, redes de conocimiento, cooperativas, alianzas estratégicas, etc.

Mediante el liderazgo con Empowerment se promueve la innovación en la organización a través de la toma de decisiones intratégicas que propician la integración y combinación de las capacidades dinámicas de la empresa. Dichas capacidades (Tecnológicas, estratégicas y organizacionales) son desarrolladas por aquellas empresas que mejoran continuamente sus procesos y productos (o innovan) para ser competitivas.

Ajustarse al ritmo del cambio significa que todos los miembros de la organización empresarial se involucran activamente en la dinámica del proceso de innovación.

4. Locus de control interno para impulsar el cambio continuo

El locus de control constituye una variable que permite una confiable predicción de la conducta futura de un sujeto en cuanto a lo que tiene que ver con la toma de decisiones. Según Durán (2001: 8), el locus de control también es conocido dentro de las teorías del aprendizaje social como la identificación de donde se origina la fuerza motivadora que lleva a los sujetos a actuar de cierta manera y no de otra ante una misma circunstancia. 
Conforme a Castro (2005), el locus de control puede ser interno ó externo. Cuando el locus de control de un individuo es externo, sus decisiones y comportamientos en la organización dependen de las circunstancias del entorno. Contrariamente, cuando el locus de control de un individuo es interno, la conducta es equilibrada e interdependiente con su entorno.

Tratándose de una situación para estimular innovaciones menores o mayores en la organización, el locus de control constituye una explicación del lugar o foco en el cual se ubica el núcleo que refuerza la realización de las conductas o acciones de los sujetos orientadas al proceso innovador, en este caso, el núcleo que refuerza la realización de conductas innovadoras se ubica en la actitud creativa del miembro de la organización y en la dirección o gerencia que se manifiesta a través de un liderazgo inspirador.

De acuerdo con la Teoría de la Innovación, en la generación de innovaciones, el núcleo que refuerza la realización de conductas innovadoras no sólo se ubica en la actitud creativa de miembro "empresario emprendedor" de la organización, también depende de implicaciones políticas, económicas y socioculturales que dinamizan tal proceso.

Conforme al enfoque teórico de la Gestión del Conocimiento y a las características del Liderazgo con Empowerment descrito en la evolución de la Teoría Gerencial, se detecta que parte del núcleo que refuerza la realización de conductas innovadoras no solo se ubica en la actitud creativa del miembro "empresario emprendedor" sino también en el miembro "seguidor emprendedor" debido a que ambos participan en el surgimiento y desarrollo de innovaciones.

Por lo tanto, la teoría de la innovación se limita a destacar las características del locus de control interno de los líderes de una organización más no de sus seguidores y además, resalta el impacto de los factores externos en el locus de control de los miembros innovadores.

De acuerdo a Schumpeter (1944; cit por García, 1989: 60), la realización de una innovación constituye una función especial, solo realizable por hombres también especiales, dotados de una visión privilegiada de los acontecimientos económicos y una dosis suficiente de intuición y "arrojo" que los impulsa a innovar, aún cuando no se puedan conocer ni anticipar todos los efectos de su acción. En tal sentido, se interpretan las características mencionadas por Schumpeter, como fuerzas motoras internas propias de un líder emprendedor.

En tal sentido la actitud de los innovadores (tanto líderes como seguidores) en la organización debe estar marcada por un alto grado de interdependencia en la toma de decisiones y comportamientos durante el proceso inventivo, la interdependencia es el tipo de fuerza motivadora que necesita estimular la gerencia para que las innovaciones ocurran de forma genuina y continua dentro de las organizaciones.

Un liderazgo con Empowerment es inspirador y permite desarrollar un alto grado de interdependencia para fortalecer la cultura innovadora de la organización empresarial.

5. Mantener la competencia McFarland, Senn y Childress (1996:161), aseguran que una empresa 
altamente competitiva es aquella capaz de dominar el cambio, siguiendo siete pasos fundamentales: hacerse".

1) Mantener un espíritu de "puede

2) Identificar a los "observadores críticos" y a los "participantes partidarios". A los primeros, conviene hacerles ver los beneficios del cambio y a los segundos, comprometerlos de inmediatos en el proceso de cambio.

3) Manejar el temor a lo desconocido y el miedo al fracaso asegurando que todos estén informados y actualizados en el "saber hacer" de los procesos.

4) Solicitar el aporte constructivo de todos, medir su progreso en el cambio, comunicar y elogiar dicho progreso.

5) Inventar maneras de reflejar el "dominio del cambio" en su visión, en sus valores, sus estrategias, su cultura y en las operaciones diarias.

6) Crear un ambiente de aprendizaje que enseñe como ser flexible y fluir con el cambio y utilizar el cambio para provecho de todos.

7) Incluir a todos en el juego del dominio del cambio, que significa "ganar ganar" para todos.

Mantener la competitividad, implica relacionar el cambio con la calidad total, el servicio superior, el mejoramiento continuo, la innovación y la educación continuada. La innovación es el dominio de todos en la compañía (Buckley, 2000: 78) y el Liderazgo con Empowerment promueve la difusión del dominio ó poder hacia toda la organización.

Una empresa necesita mejorar constantemente (es decir innovar) su estrategia y su intrategia para mantener su posición competitiva (Cardona, 2001:19).
El Empowerment como estilo de liderazgo permite a los jefes de las organizaciones concentrarse en necesidades más amplias, como la formulación de nuevas visiones, la determinación de estrategias e intrategias y en prioridades referentes al entrenamiento de equipos que configuran una cultura sustentada en la excelencia.

Según McFarland, Senn y Childress (1996:39), los vientos de la competencia están soplando en la mayor parte de las compañías, las cuales enfrentan enormes presiones. Esto resulta cierto aún para compañías que tradicionalmente fueron inmunes. Por ejemplo, gracias a las privatizaciones en otros países, las compañías de teléfonos, que eran clásicos monopolios regionales, son ahora competidores globales. A esta intensa competencia global deben agregarse los años de desregulación en industrias como aerolíneas, que se esfuerzan por competir y las otrora protegidas compañías de servicios, que ahora deben proporcionar acceso a sus redes de gas, de electricidad y de teléfonos a competidores externos.

En tal sentido, también se destaca que la globalización esta obligando a las organizaciones a aprender, a innovar y a operar más eficientemente con más calidad y excelencia para poder competir con éxito en el mercado mundial.

Para alcanzar estándares de desempeño de clase mundial, las organizaciones tienen que producir una fuerza de trabajo más inteligente mejorando la educación, respetando y honrando la diversidad de los individuos, y habilitándolos en su búsqueda por maximizar su talento, su creatividad y todo su potencial, esto permitirá crear valor y mantener la competencia. 
6. Optimismo, creatividad y colaboración cruzada

No es posible todavía en muchas organizaciones liberar el potencial de su gente y obtener lo mejor de cada uno de sus miembros debido a la cultura y al estilo de liderazgo existente en combinación con la rigidez de una estructura jerárquica tradicional. Sin embargo, dada la complejidad del "juego del cambio" puede implementarse mejor el Empowerment mediante un equipo funcional y organizacional de tipo cruzado, donde sus miembros constituyan una red cocreativa de visión, de valores, proyectos e ideas (McFarland, Senn y Childress, 1996:80).

Lo anterior significa que el Liderazgo con Empowerment promueve la organización de equipos funcionales de acción cuya capacidad les permite a sus miembros colaborar libremente en toda clase de retos empresariales. Con frecuencia, estos equipos se constituyen de manera temporal para llevar a cabo una misión específica. Los miembros del equipo tienen las herramientas apropiadas para la tarea. La posición, el nivel, el título y la antigüedad son todos irrelevantes debido a que los miembros contribuyen con su talento y sus destrezas para completar la tarea.

La idea básica que promueve la característica del optimismo, creatividad y colaboración cruzada, consiste en romper con las estructuras tradicionales dentro de la organización para favorecer los procesos de innovación, invirtiendo la pirámide organizacional con la finalidad de que todos sus miembros participen armoniosa y activamente como impulsores de dicho proceso.
La dinámica del proceso innovador es compleja debido a sus características y la naturaleza de las relaciones entre los factores que la determinan. Desde el enfoque economicista de la innovación, tales factores son: las políticas externas e internas, las relaciones de mercado, las características geográficas, el cambio técnico y las actividades de investigación y desarrollo I\&D. Sin embargo, conviene destacar que desde la perspectiva gerencial, un aspecto facilitador del fenómeno de la innovación dentro de las organizaciones se refiere al ambiente organizacional propiciado por el liderazgo. La creación de este ambiente organizacional, debe estar orientada hacia el logro de la participación auto motivada de los miembros, quienes deben estar convencidos que el Empowerment como modelo de liderazgo organizacional puede ser fácil, natural y desde luego divertido, pues sólo con estas actitudes optimistas se fomentará un entorno creativo e innovador en la organización empresarial.

\section{Conclusiones}

El modelo de Liderazgo con Empowerment como promotor de la innovación, constituye en la organización empresarial un medio para la creación de climas innovadores, donde la dirección estimula la democratización de la información, el desarrollo de los colaboradores, la difusión de la creatividad como valor y la receptividad a las sugerencias.

De acuerdo a los resultados de este estudio, es posible describir el Modelo de Liderazgo con Empowerment como promotor de la innovación bajo la 
consideración de las siguientes características: 1. Cultura Empowerment con valores innovativos, 2. Confianza y compromiso hacia la organización empresarial, 3. La intrategia como instrumento para la unidad, 4. El locus de control interno para impulsar el cambio continuo, 5. Mantener la competencia y 6 . Optimismo, creatividad y colaboración cruzada.

Manteniendo estas características, el liderazgo organizacional puede identificar de forma colaborativa focos de innovación, analizar y definir problemas en equipo y en consenso, atender a la deseada alineación y sinergia, evaluar las propuestas innovadoras, poner en práctica ideas valiosas, reconocer los esfuerzos creativos y consolidar la experiencia innovadora, para contribuir al éxito en la organización empresarial.

Muchas organizaciones han optado por implementar el enfoque de liderazgo con Empowerment para adaptarse a los cambios del entorno y promover una cultura de innovación; no obstante, no se ha manifestado en ellas un cambio estructural que les permita desarrollar una productiva dinámica de innovación quizás por no cumplir con alguna de las características del perfil señalado, por lo que además, podría considerarse que son organizaciones parcialmente innovadoras.

Como aporte especial de este trabajo, es importante hacer notar que se produjo un punto de encuentro conceptual entre la Teoría de la Innovación, el Enfoque de la Gestión del Conocimiento y la Teoría Gerencial, al lograr construir el perfil promotor de innovación del Modelo de Liderazgo con Empowerment. Este punto de encuentro consiste en que todas estas perspectivas teóricas asumen al liderazgo como un determinante de la innovación.

Al intentar vincular la Teoría de la Innovación con la Teoría Gerencial, se encontraron varias diferencias conceptuales en relación al tema del liderazgo organizacional para la innovación: 1. El primero de estos cuerpos teóricos no considera al "seguidor emprendedor" como determinante del proceso innovador, sino solamente a los "empresarios emprendedores" a quienes caracteriza como "hombres especiales" dotados de una visión privilegiada de los acontecimientos económicos y una dosis suficiente de intuición y arrojo que los impulsa a innovar con la finalidad de dar valor comercial a las ideas.

2. Por su parte, la Teoría Gerencial en el marco de la Gestión del Conocimiento y el Modelo de Liderazgo con Empowerment, explica conceptualmente cómo es posible aprovechar las ideas explotando recursos, generando capacidades y desarrollando competencias en la organización a partir de las aportaciones de todos sus miembros, centrándose en el valor de uso de las ideas, lo cual constituye el eslabón que permite relacionar las variables "Liderazgo Empowerment" y "proceso de innovación"

\section{Referencias Bibliográficas}

\section{Buckley, J. (2000). Cómo crecer con ventaja competitiva. El valor real de la tec- nología. Colombia. Mc Graw Hill.}

Cardona S. (2001). "Intrategia: una dimensión básica de la cultura empresarial”. En Álvarez de Mon, Cardona S., Chinchilla, A., Millar, Pérez, L., Pin, A., Poel- 
mans, Rodríguez, L., Rodríguez, P., Torres (2001). Paradigmas de Liderazgo. España. McGraw-Hill Interamericana de España, S.A.

Caires, G. (2003). Evolución de la Disciplina de "Gestión Tecnológica". Sinopsis del artículo con el mismo artículo. SELA Sistema Económico Latinoamericano Caracas, Venezuela.

Castro, E. (2005). Apuntes de clase sobre Liderazgo organizacional, en el Seminario "Liderazgo organizacional desde una perspectiva teóricapráctica para el logro de una gestión efectiva", Doctorado de Ciencias Humanas, Facultad de Humanidades y Educación, La Universidad del Zulia, Maracaibo, Venezuela.

De Geus, A. (1997). The living company. Harvard Business Review. Marzo/abril.

Durán R., T. El constructo locus de control en la toma de decisiones educativas. En revista PAEDAGOGIUM México, Centro de Investigación y Asesoría Pedagógica, S.C. Año 1, núm. 3 ene-feb 2001. pp. 8-11.

García, H. (1989). Política e innovación tecnológica: Perspectivas económicas. Caracas, Venezuela. Monte Ávila Editores.

INNOVA (2003). Gestâo \& Tecnología. Boletín do núcleo de política e gestâo tecnologica da universidade de Sâo Paulo. Año X-№ 36 Outubro/Novembro/Dezembro de 2003.

Kockelmans, J. (1975): "Toward an interpretative or hermeneutic social science". En: Graduate Faculty Philosophy
Journal. Tomo 5, № 1. Pp. 73/96. Citado por: Martínez M. (1996): Comportamiento Humano-Nuevos Métodos de Investigación. México. Editorial Trillas.

McFarland, L.; Senn, L.; Childress, J. (1996). Liderazgo para el siglo XXI. Colombia. McGraw-Hill Interamericana, S.A.

Nelson, R.; Winter, S. (1982). An Evolutionary Theory of Economic Change. Harvard University Press, Cambridge, M.A.

Pérez, C. (1989). Technical Change, competitive restructuring and institutional reform in developing countries, Banco Mundial, strategic Planning and Review Department Paper, № 4, diciembre.

Pfeffer, J. (1998). The Human Equation. Boston. Harvard Business Scool Press.

Quigley, J. (1997). Visión. Como la desarroIlan los líderes, la comparten y la sustentan. Colombia. Mc Graw Hill.

Rosenberg, N. (1979). Economía del cambio tecnológico. Selección 31. Trimestre Económico. México. Fondo de Cultura Económica.

Schumpeter, J. (1944). Teoría del desenvolvimiento económico. F.C.E. México.

Schmoockler, J. (1962). Fuentes económicas de la actividad inventiva, Journal of economics history. Marzo. Pp. 1-20.

Usher (1955). Technical change and capital formation. Capital Formation and Economic Growth, Oficina Nacional de Investigación Econónmica. Pp. 523-550. 